\title{
THERMAL-PROCESSING OF CARBON DUST AROUND A COOL STAR
}

\section{K. A. K. Gadallah}

Department of Astronomy \& Meteorology, Faculty of Science, Al-Azhar University, Nasr City, PO Box 11884 Cairo,Egypt, email:Kamel.Gadallah71@gmail.com

\section{ABSTRACT:}

Thermal-processed carbonaceous materials could emulate the evolution of the carbonaceous dust which settles in regions around a cool star in a proto-planetary nebula (PPN). Spectroscopically, this evolution has been shown through the variation in the strength of the aliphatic $(-C-H$ stretching mode at the $3.42 \mu \mathrm{m})$ band relative to those of the aromatic $(=C-H$ mode at the $3.3 \mu \mathrm{m})$ band. Experimentally, the laser ablation technique was used to produce the Hydrogenated Amorphous Carbon (HAC) samples as analogs of comic carbon dust. The IR-laboratory data of these samples shows that the strength of these two bands varies reversely to each other as HAC transforms from the aliphatic to aromatic structure with heating. The temperature, required to cause this transformation within HAC, depends on its hydrogen content. Hence, the trend of this variation is also in relevance to the hydrogen content within HAC. This trend shows the variation from the aliphatic to aromatic structure in dust grains in regions within the PPN IRAS 22272+5435. In which, it is expected that dust grains may be heated in the past in the warm region near the star.

Keywords: Comic carbon dust, IR spectroscopy, Planetary nebulae: PPN IRAS 22272+5435.

\section{INTRODUCTION}

The outflows, coming from cool carbon stars into the interstellar medium (ISM), contain solid carbonaceous materials, which are formed from cooling C-rich gas. These materials are extremely important components of dust grains in the diffuse ISM (Greenberg and Li, 1999; Ehrenfreund and Charnley, 2000). Several forms of hydrocarbon materials with different fractions of $\mathrm{sp}^{1}, \mathrm{sp}^{2}$ and $\mathrm{sp}^{3}$ hybridizations could be graphite, diamond, polycyclic aromatic hydrocarbons (PAH), amorphous carbon (AC), or hydrogenated amorphous carbon (HAC). These, aliphatic structures are present in the ISM in our own and other galaxies, as evidenced by observations of vibration absorption features centered at $3.4 \mu \mathrm{m}$ (-C-H stretching) and at 6.85 and $7.25 \mu \mathrm{m}$ (-C$\mathrm{H}$ deformation or bending modes). In the Milky Way, hydrocarbons have been detected along lines of sight in the local diffuse ISM within 3 kpc of the Sun (Pendleton et al., 1994) and toward the galactic center (e.g., Chiar et al., 2000, 2002). Similar spectroscopic features of hydrocarbons are also observed in dusty Seyfert and ultraluminous infrared galaxies (e.g., Spoon et al., 2004; Imanishi et al., 2006). In the study by Dartois and Muñoz-Caro (2007), extragalactic spectra by Spitzer IRS reveal that HAC materials are common in the ISM of embedded luminous-infrared galaxies. Based on the depth of the corresponding deformation features at 6.85 and $7.25 \mu \mathrm{m}$, the best candidate material is likely to be the HAC material (Pendleton and Allamandola, 2002). In a spectroscopic technique, the structure of HAC materials in a mixture of the aliphatic and aromatic carbon has been described recently in relevance to the strength of the midinfrared features (Jones, 2012a,b,c; Gadallah et al., 2012, 2013).

In warm regions flooded by the galactic superwind in the nearby edge-on starburst galaxy (M82), the thermal process of the cosmic carbon dust shows an important impact on its structure. The fraction of the aliphatic to the aromatic feature increases with the distance from the galactic center (Yamagishi et al., 2012). The observed spectra of extremely carbonrich proto-planetary nebula (PPN) IRAS 22272 +5435 (Goto et al., 2003) show transformation from the aliphatic $-\mathrm{C}-\mathrm{H}$ to aromatic $=\mathrm{C}-\mathrm{H}$ stretching mode within dust grains in the circumstellar shells. Despite this PPN is not hot enough to ionize the surrounding the asymptotic giant branch (AGB) ejecta and is not yet characterized by the hard UV radiation during its short evolutionary phase $\left(10^{3} \mathrm{yr}\right)$ between AGB and PN phases, this transformation reveals the thermal process of very small carbon dust grains. This processing might occur either by the stochastic heating by absorbing UV photons in the circumstellar shells around a cool star in this PPN or already occurred in the past when the grains were formed in a warm 
region near the star. The last assumption is mostly expected (Goto et al., 2003) because the absence of hydrogen recombination lines at $3.74 \mu \mathrm{m}$ (Pf) and 4.05 $\mu \mathrm{m}(\mathrm{Br})$ indicates that there is no HII region at the center of this nebula. Additionally, the hydrocarbon emission features are not seen near the central star. This transformation takes place during environmental changes of increasing stellar temperature Goto et al. (2000). Experimentally, these authors found that the quenched carbonaceous composite (QCC) requires about $823 \mathrm{~K}$ to reveal this transformation in its structure. In the current work, the required temperature that causes this transformation in HAC will be discussed in relevance to hydrogen content.

\section{HAC-MID IR BANDS}

In astrophysical approaches, laboratory analogs of carbon dust are under discussion to confirm the idea of amorphous carbon as a component of cosmic dust (e. g., Schnaiter et al., 1998; Jäger et al., 1999, 2008; Furton et al., 1999; Gadallah et al., 2011, 2012). The analyses given by these analogs have been able to construct interstellar amorphous carbon materials. The properties of these materials could explain some of the astronomical features. The vibrational properties of the HAC material can be inferred from the IR spectral signatures (absorption/emission) of the organic component of interstellar dust grains. Such these signatures are the aliphatic C$\mathrm{H}$ stretching band at $3.4 \mu \mathrm{m}$ and the $\mathrm{C}-\mathrm{H}$ bending bands at both 6.85 and $7.25 \mu \mathrm{m}$. The $3.4 \mu \mathrm{m}$ band includes sub-features at about 3.38, 3.42, 3.48 and $3.51 \mu \mathrm{m}$ (Gadallah et al., 2013). These sub-features represent the asymmetric mode of $\mathrm{CH}_{3}$ and $\mathrm{CH}_{2}$, and the symmetric mode of $\mathrm{CH}_{3}$ and $\mathrm{CH}_{2}$, respectively. The aliphatic stretching and bending bands of HAC can be influenced (reduced or probably removed) in the laboratory by thermal treatments (Mennella et al., 1997; Gadallah et al., 2013) and by UV irradiation (Mennella et al., 2001; Murnoz-Caro et al., 2001; Gadallah et al., 2011, 2012). There is a similarity of their evolution seen in astronomical observations. The absence of the aliphatic bands in circumstellar shells around AGB and post AGB stars indicates that their carrier is a product which is formed during the transition from AGB to PN phase (Duley, 2000; Jones et al., 1990). In the observed spectra toward the dense and diffuse interstellar regions, the remarkable variations in the strength of these bands are observed (Chiar et al., 2000). All bands in the mid-IR region of the experimental IR data of various analogs are in coincidence with those in the ISM (Chiar et al., 2000; Pendleton and Allamandola, 2002). Consequently, HAC materials are considered for being an important component in the circumstellar and interstellar environments.

\section{Experiments and FTIR measurements}

Samples of HAC in a nano-sized scale were produced by the laser ablation technique of a rotating graphite target in a quenching gas atmosphere $\left(\mathrm{He} / \mathrm{H}_{2}\right)$ at pressure of 4.5 mbar. Physical conditions, used for precipitation on different substrates, are given in Table 1. In that technique, a Nd:YAG laser with a wavelength of $532 \mathrm{~nm}$ was used. A laser power of $2.8 \times 10^{10} \mathrm{Wcm}^{-2}$ and another with approximately two orders of magnitude higher were used to produce samples 1 and 2 , respectively. More details about the estimated and adapted laser power are given in Gadallah et al. (2011). The laser beam can vaporize very small quantities of graphite as intense plasma in which the temperature is about $4000 \mathrm{~K}$ (Jäger et al., 2008) but, using higher laser power, the temperature becomes higher than $4000 \mathrm{~K}$ (Iida and Yeung, 1994). Collisions between the evaporated carbon components with the quenching gas in a supersonic expansion of the hot plasma cause the condensation of carbon particles. In case of using the lower laser power, the deposition rate was about $1.6 \pm 0.4 \mathrm{~nm} / \mathrm{min}$ but, at using the higher value, it increases to be about $2.5 \pm 0.5 \mathrm{~nm} / \mathrm{min}$. With increasing the laser power to the maximum value, the laser beam which irradiates the graphite target was focused on a very smaller spot $(0.3$ $\mathrm{nm}$ in diameter). Samples were deposited in a separate chamber (substrate chamber, for details please see Figure 1 in Jäger et al. (2008)) at a pressure of $(5 \pm 3) \times 10^{-6}$ Torr.

Using an electrical heating system mounted in the chamber of the deposition in high vacuum (approximately $10^{-6}$ Torr), the sample 1 was heated at 473, 573, 623 and $703 \mathrm{~K}$ while sample 2 was heated at 600 and $703 \mathrm{~K}$. A thermocouple connected with the sample's holder inside the 
substrate chamber was used to measure the temperature of the sample. In this system, the temperature was controlled to rise slowly up to 323 $\mathrm{K}$ and remained constant for at least one hour to remove chemical adsorbents by pumping system. The temperature was also kept constant at $473 \mathrm{~K}$ for two hours or more to purge some radicals of hydrocarbons which were present inside the chamber due to heating.

Because the Fourier Transformation IR spectroscopy (FTIR) is based on the vibrations of the atoms of a molecule, it can be used to study the chemical bonding of hydrogen and its concentration in a HAC material. The possible vibrational modes of HAC materials (or carbon soot) had been tabulated by e.g. Jäger et al. (2008). The IR transmission measurements were taken in-situ by using the FTIR spectrometer (Bruker 113v). During the measurements, a blank substrate was used as the reference for the transmission spectrum for each sample. A base-line correction of the experimental spectra was done in order to subtract the continuum absorption.

Table 1: Physical conditions and analytical results of HAC thin films.

\begin{tabular}{ccccccc}
\hline Samples $^{(1)}$ & $\begin{array}{c}\text { thickness } \\
(\mathrm{nm})^{(2)}\end{array}$ & $\mathrm{He}: \mathrm{H}_{2}$ & substrate & $\begin{array}{c}\mathrm{Fl} \\
\left(\left(\mathrm{Wcm}^{-2}\right)\right.\end{array}$ & $\mathrm{H} / \mathrm{C}$ & $\mathrm{T}_{\mathrm{r}}(\mathrm{K})$ \\
\hline 1 & 117 & $50: 30$ & $\mathrm{KBr}$ & $2.0 \times 10^{8}$ & 0.60 & $\leq 703$ \\
2 & 93 & $90: 10$ & $\mathrm{CaF}_{2}$ & $2.8 \times 10^{10}$ & 0.78 & $>703$ \\
\hline
\end{tabular}

(1) Samples were produced at pressure of 4.5 Torr at the room temperature; (2)Thicknesses were given by a microbalance based on an assumed mass density of 1.55 $\mathrm{gcm}^{-3}$.

When carbon and hydrogen atoms are brought together, the $=\mathrm{C}-\mathrm{H}$ and $-\mathrm{C}-\mathrm{H}_{2,3}$ groups are formed. These groups are responsible for the observed absorption bands of such compounds near 3.3 $\mu \mathrm{m}$ and $3.4 \mu \mathrm{m}$, respectively. The strength of the aliphatic $3.4 \mu \mathrm{m}$ band is very stronger than those of the aromatic $3.3 \mu \mathrm{m}$ band. With heating, the HAC material shows aromatization structure, in which $\mathrm{sp}^{2}$ sites dominate its electronic structure resulting in strong aromatic $=\mathrm{C}-\mathrm{H}$ stretching band at $3.3 \mu \mathrm{m}$ but the aliphatic bands around $3.4 \mu \mathrm{m}$ vanish (Gadallah et al., 2013). In addition to the production conditions, the electronic and atomic structure of HAC could be controlled by the thermal processing. Finally, the spectro- scopic properties of HAC depend mainly on the hydrogen content.

\section{RESULTS}

\subsection{Experimental results}

The atomic and electronic structures of HAC are analytically explained by the spectroscopy and transmission electronic microscope (TEM) techniques in the previous work by Gadallah et al. $(2011,2012,2013)$. The morphology of HAC shows that a typical appearance of strongly bent disordered-graphene layers and small fullerenelike carbon grains are observed. In the production conditions of these materials, any change in the laser power leads to a variation in the electronic structure of the produced carbonaceous nanoparticles as a result of the change in the vibrational temperature in the condensation. As listed in Table 1 , sample 1 was produced by using low laser power $\left(2 \times 10^{8} \mathrm{Wcm}^{-2}\right)$, which is close to that in Jäger et al. (2008). For sample 2, a value with approximately two orders of magnitude higher $\left(2.8 \times 10^{10} \mathrm{Wcm}^{-2}\right)$ was used. The estimated hydrogen content $(\mathrm{H} / \mathrm{C}$ ratio) of the samples was spectroscopically derived from the integrated area under the C-H stretching bands around 3.4 $\mu \mathrm{m}$ (Angus and Jansen, 1988; Gadallah et al., 2011). For sample 1 the hydrogen content is 0.60 while, for the sample 2, it is higher to become 0.78 because of the higher plasma-temperature due to increasing the laser power. In this table, the last column represents approximately the required temperature $\left(\mathrm{T}_{\mathrm{r}}\right)$ that causes the transformation from aliphatic to aromatic structures. At which most of the $\mathrm{sp}^{3}$ aliphatic bonding between carbon and hydrogen atoms is broken while the $\mathrm{sp}^{2}$ aromatic bonding between these atoms becomes the dominant structure. With heating of HAC, the length of the garphene layers increases due to the release of hydrogen atoms leading to bigger aromatic areas, which could be precursors of PAH (Gadallah et al., 2013). The hydrogen atoms are hanging on the edge of these areas. For sample 1, having low value of the hydrogen content, it seems that the aliphatic structure vanishes with heating temperature, which is limited from $>623 \mathrm{~K}$ to $\leq 703 \mathrm{~K}$ as shown in Figure 1. For sample 2 having a higher value of the hydrogen content, its spectrum is still showing weak ali- 
phatic structures at $703 \mathrm{~K}$ therefore higher temperature than $703 \mathrm{~K}$ is required to remove completely all aliphatic structures. The mid-IR spectra of all samples are shown in Figure 1. Spectra show aromatic and aliphatic structures at 3.3 and $3.4 \mu \mathrm{m}$ bands, respectively. Carbon atoms are bonded with hydrogen atoms in different functional groups, such as in aromatic $=\mathrm{C}-\mathrm{H}$, and in saturated aliphatic $-\mathrm{CH}_{3},-\mathrm{CH}_{2}$, or - $\mathrm{CH}$ groups. It is shown that, for the highly focused laser power, the mass absorption coefficient $(\kappa)$ is higher than that in case of using the lower value of the laser power.

In the study by Gadallah et al. (2013), TEM images of HAC heated up to $703 \mathrm{~K}$ shows an elongation of the average length of the graphene layers from $7.2 \AA$ to $20 \AA$. In this study, more details are given about aromatization of HAC with heating. There is an increase in the aromatic rings or the aromatic skeleton ( $\mathrm{sp}^{2}$ hybridization)

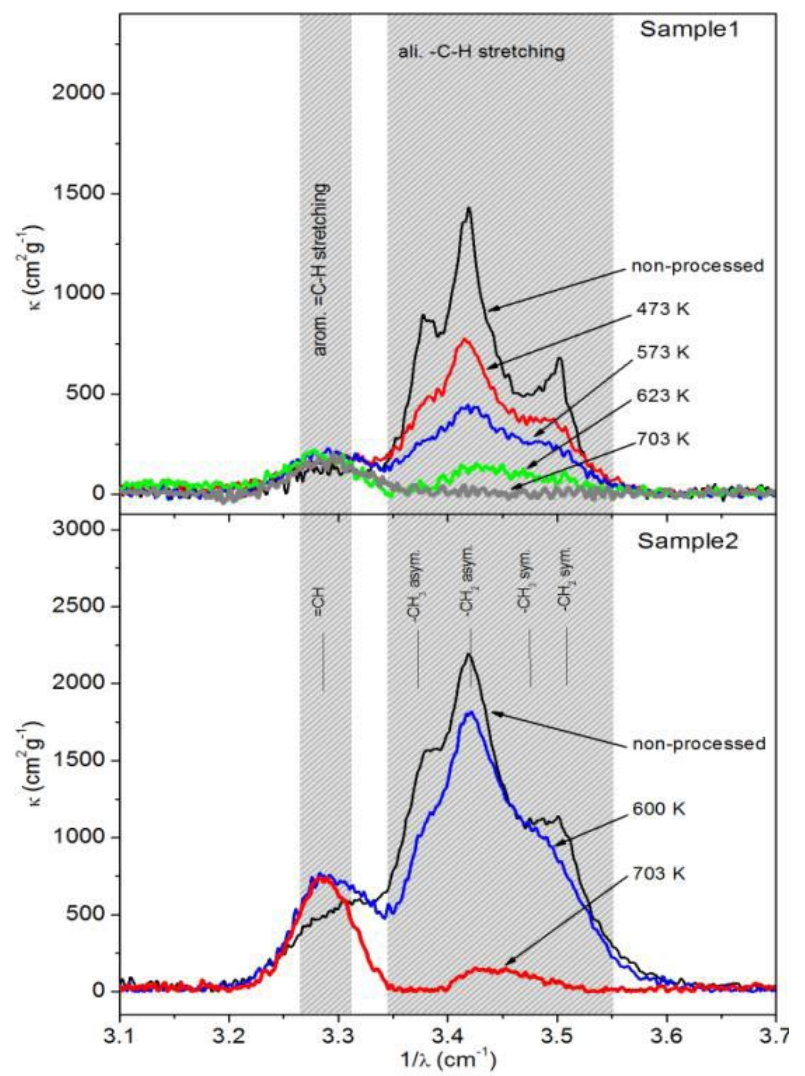

Figure 1: The spectra of $\mathrm{HAC}$ samples produced by the laser ablation technique. Samples 1 was produced by using low laser power $\left(2 \times 10^{8} \mathrm{~W} \mathrm{~cm}^{-2}\right)$ while sample 2 was produced by using high laser power $\left(2.8 \times 10^{10} \mathrm{~W} \mathrm{~cm}^{-2}\right)$. Sampel 1 was heated at $473,573,623$ and $703 \mathrm{~K}$ while sample 2 was heated at 600 and $703 \mathrm{~K}$. due to heating. Spectra of heated-HAC show spectroscopic transformation from the aliphatic $-\mathrm{C}-\mathrm{H}$ to aromatic $=\mathrm{C}-\mathrm{H}$ stretching, as shown in Figure 1. The aliphatic structure of sample 1 having low hydrogen content, disappears mostly at temperature of $703 \mathrm{~K}$. On the other hand, the spectrum of the sample 2, possessing high hydrogen content, still shows some of the aliphatic bands in the $3.4 \mu \mathrm{m}$ plateau. It is obvious that this transformation is attributable to how much the hydrogen content is within HAC. Another comparative carbonaceous material is the QCC, which was produced by the technique of the hydrogen plasma deposition (Goto et al., 2000). In this material, the increase of the aromatic rings which lead to the formation of carbon onion-like spherules is directly cause the transformation from the aliphatic to aromatic $\mathrm{C}-\mathrm{H}$ structure. Goto et al. (2000) found that it takes place for the QCC at temperature of $823 \mathrm{~K}$, which is higher than the required temperature $(>623 \mathrm{~K}$ to $\leq$ $703 \mathrm{~K}$ ) for HAC having approximately hydrogen content of 0.6 .

\subsection{Astrophysical implication}

For the PPN IRAS $22272+5435$, the IR emissions have been observed (Goto et al., 2003) in a range from 600 to $1300 \mathrm{AU}$ (NE3 to NE7, respectively) from the central star at the northeast sector of the nebula. These emissions represent the aromatic structure at the $3.3 \mu \mathrm{m}$ band and the aliphatic structures around the $3.4 \mu \mathrm{m}$ band of

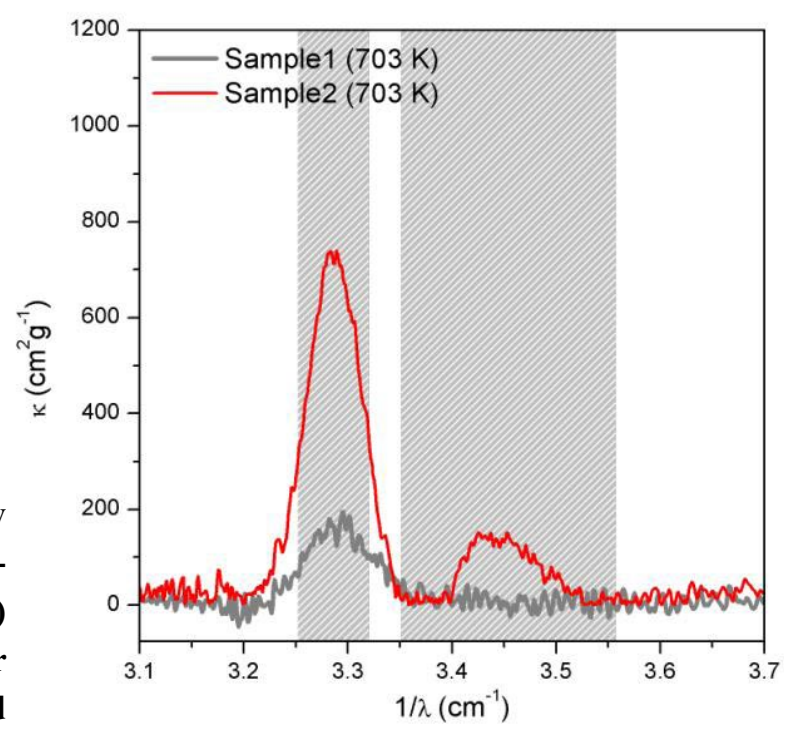

Figure 2: FTIR spectra of heated HAC (sample 1 and 2) at $703 \mathrm{~K}$. 
hydrocarbon dust. Goto et al. (2003) found that the ratio of the aliphatic band at $3.4 \mu \mathrm{m}$ to the aromatic band at $3.3 \mu \mathrm{m}$ varies inversely with the distance from the central star. Spectra shown in Figure 3 demonstrate the reversal variation in the strength of the aliphatic bands with those of the aromatic band of this nebula (left panel) in coincidence with those of the laboratory spectra (right panel) resulting from the thermal processing of HAC materials. For HAC materials having approximately hydrogen content of 0.6 , the temperature required to cause the transformation from aliphatic to aromatic structures is approximatelv $703 \mathrm{~K}$. It is lower than what Goto et al.

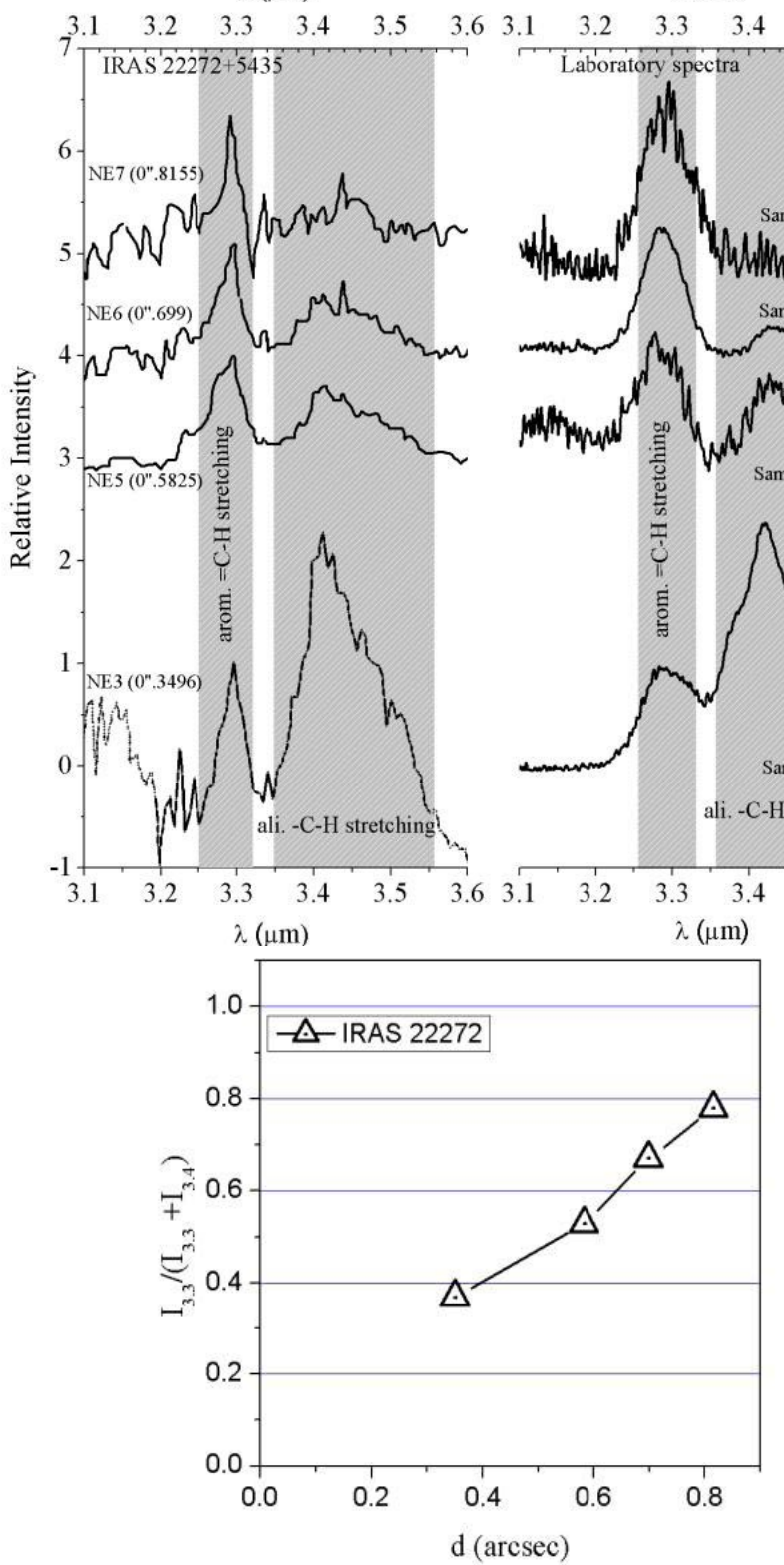

(2000) found (823 K) for QCC.

In Figure 3, the intensities of the aromatic band $\left(\mathrm{I}_{3.3}\right)$ at $3.3 \mu \mathrm{m}$ and of the aliphatic band $\left(\mathrm{I}_{3.4}\right)$ at $3.4 \mu \mathrm{m}$ vary conversely to each other with the distance in a range of 600-1300 AU (0.3496$0.8155 \mathrm{arcsec}$ ) form the central stars of the PPN IRAS $22272+5435$ (left panel). A similar variation of these intensities is also observed in the laboratory data of HAC with the heating temperature (right panel). Analytically, the trends of the intensity of the aromatic relative to those of the total (aromatic and aliphatic) are shown in Figure 4 versus the distance from the central
Figure 3: The aliphatic and aromatic C-H stretching bands of experimental data of samples 1 (623 K), 2 (703 $\mathrm{K})$ and 3 (original and 703 K) compared with those of IRAS 22272+5435 (Goto et al., 2003).

Figure 4: The intensity of the aromatic relative to those of the total (aromatic and aliphatic) versus the distance from the central stars of the PPN IRAS 22272+543 (left panel) and versus the temperature for the laboratory data (right panel). 
stars of the PPN IRAS $22272+543$ (left panel) and versus the temperature for the laboratory data (right panel). These trends show the similar transformation from aromatic to aliphatic structures in relevance to the distances from the cool central star and to a range of temperatures above $500 \mathrm{~K}$. It is shown from the laboratory data that $\operatorname{Tr}$ making this transformation depends on the hydrogen fraction within the hydrocarbon dust where the hydrocarbon materials are graphitized by annealing temperature in the ISM. Finally, as much as the hydrogen is within the carbonaceous material in the warm region around the star, the required temperature is higher to cause the transformation from the aliphatic $-\mathrm{C}-\mathrm{H}$ to aromatic $=\mathrm{C}-\mathrm{H}$ stretching modes.

\section{CONCLUSION}

Transformation from the aliphatic -C-H at 3.4 $\mu \mathrm{m}$ to aromatic $=\mathrm{C}-\mathrm{H}$ at $3.3 \mu \mathrm{m}$ stretching mode is observed within a cold region in the PPN IRAS $22272+5435$. The structure of carbonaceous dust grains could be modified once these grains passed though a warm interstellar region. Experimentally, the thermal process of HAC leads to the aromatization by elongating the graphene layer. From the laboratory data, the required temperature, at which this transformation takes place, is related to the hydrogen content. As the hydrogen content increases within HAC, the required temperature is higher. This temperature $(703 \mathrm{~K})$ is lower than that for QCC $(823 \mathrm{~K}$, Goto et al., 2000), in which the thermal process leads to the increase of the aromatic rings leading to the carbon onion-like structure.

Acknowledgment. I am very grateful to Harald Mutschke and Cornelia Jäger, in Jena at Friedrich Schiller University Jena, Germany, for allowing me to achieve these experiments.

\section{REFERENCES}

Angus, J. C. and Jansen, F.: 1988, JVST 6, 1778

Chiar, J. E., Peeters, E., and Tielens, A. G. G. M.: 2002, ApJ 579, L91

Chiar, J. E., Tielens, A. G. G. M., Whittet, D. C. B., Schutte, W. A., Boogert, A. C. A., Lutz, D., van Dishoeck, E. F., and Bernstein, M. P.: 2000, ApJ 537, 749

Dartois, E. and Mu noz-Caro, G. M.: 2007, A\&A 476, 1235

Duley, W. W. 2000, ApJ, 528, 841
Ehrenfreund, P. and Charnley, S. B.: 2000, ARA\&A 38, 427

Furton, D. G., Laiho, J. W., and Witt, A. N.: 1999, ApJ 526, 752

Gadallah, K. A. K., Mutschke, H., and Jäger, C.: 2011, A\&A 528, A56

Gadallah, K. A. K., Mutschke, H., and Jäger, C.: 2012, A\&A 544, A 107

Gadallah, K. A. K., Mutschke, H., and Jäger, C.: 2013, A\&A 554, A12

Goto, M., Gaessler, W., Hayano, Y., Iye, M., Kamata, Y., Kanzawa, T., Kobayashi,

N., Minowa, Y., Saint-Jacques, D. J., Takami, H., Takato, N., and Terada, H.: 2003, ApJ 589, 419

Goto, M., Maihara, T., Terada, H., Kaito, C., Kimura, S., and Wada, S.: 2000, A\&AS 141, 149

Greenberg, J. M. and Li, A.: 1999, ASR 24, 497

Iida, Y. and Yeung, E. S.: 1994, Appl. Spectrosc. 48(8), 945

Imanishi, M., Dudley, C. C., and Maloney, P. R.: 2006, ApJ 637,114

Jäger, C., Henning, T., Schlogl, R., and Spillecke, O.: 1999, J. Non-Cryst. Solids. 258, 161

Jäger, C., Mutschke, H., Henning, T., and Huisken, F.: 2008, ApJ 689, 249

Jones, A. P.: 2012a, A\&A 540, A1

Jones, A. P.: 2012b, A\&A 540, A2

Jones, A. P.: 2012c, A\&A 542, A98

Jones, A. P., Duley, W. W., and Williams, D. A.: 1990, QJRAS 31, 567

Mennella, V., Baratta, G. A., Colangeli, L., Palumbo, P., Rotundi, A., Bussoletti, E., and Strazzulla, G.: 1997, ApJ 481, 545

Mennella, V., Mu noz Caro, G. M., Ruiterkamp, R., Schutte, W. A., Greenberg, J.

M., Brucato, J. R., and Colangeli, L.: 2001, A\&A 367, 355

Muñoz-Caro, G. M., Ruiterkamp, R., Schutte, W. A., Greenberg, J. M., and Mennella, V.: 2001, A\&A 367, 347

Pendleton, Y. J. and Allamandola, L. J.: 2002, ApJS 138, 75

Pendleton, Y. J., Sandford, S. A., Allamandola, L. J., Tielens, A. G. G. M., and

Sellgren, K.: 1994, ApJ 437, 683

Schnaiter, M., Mutschke, H., Dorschner, J., Henning, T., and Salama, F.: 1998, ApJ 498, 486

Spoon, H. W. W., Armus, L., Cami, J., Tielens, A. G. G. M., Chiar, J. E., Peeters,

E., Keane, J. V., Charmandaris, V., Appleton, P. N., Teplitz, H. I., and

Burgdorf, M. J.: 2004, ApJS 154, 184

Yamagishi, M., Kaneda, H., Ishihara, D., Kondo, T., Onaka, T., Suzuki, T., and Minh, Y. C.: 2012, A\&A 541, A10 